\title{
Impact of Ground Effect on Airplane Lateral Directional Stability during Take-Off and Landing
}

\author{
M. Sereez, N. Abramov, M. G. Goman \\ De Montfort University, Leicester, UK \\ Email: mgoman@dmu.ac.uk
}

How to cite this paper: Sereez, M., Abramov, N. and Goman, M.G. (2018) Impact of Ground Effect on Airplane Lateral Directional Stability during Take-Off and Landing. Open Journal of Fluid Dynamics, 8, 1-14.

https://doi.org/10.4236/ojfd.2018.81001

Received: January 29, 2018

Accepted: March 6, 2018

Published: March 9, 2018

Copyright ( 92018 by authors and Scientific Research Publishing Inc. This work is licensed under the Creative Commons Attribution International License (CC BY 4.0).

http://creativecommons.org/licenses/by/4.0/

\begin{abstract}
Computational simulations of aerodynamic characteristics of the Common Research Model (CRM), representing a typical transport airliner are conducted using CFD methods in close proximity to the ground. The obtained dependencies on bank angle for aerodynamic forces and moments are further used in stability and controllability analysis of the lateral-directional aircraft motion. Essential changes in the lateral-directional modes in close proximity to the ground have been identified. For example, with approach to the ground, the roll subsidence and spiral eigenvalues are merging creating the oscillatory Roll-Spiral mode with quite significant frequency. This transformation of the lateral-directional dynamics in piloted simulation may affect the aircraft responses to external crosswind, modify handling quality characteristics and improve realism of crosswind landing. The material of this paper was presented at the Seventh European Conference for Aeronautics and Space Sciences EUCASS-2017. Further work is carried out for evaluation of the ground effect aerodynamics for a high-lift configuration based on a hybrid geometry of DLR F11 and NASA GTM models with fully deployed flaps and slats. Some aspects of grid generation for a high lift configuration using structured blocking approach are discussed.
\end{abstract}

\section{Keywords}

CFD, High-Lift Configuration, Ground Effect, Airplane Stability, Lateral-Directional Modes

\section{Introduction}

According to statistics of fatal accidents worldwide for commercial Jet Fleet 
during the period 2006-2015 presented by Boeing Company Ltd the number of fatalities during landing due to Abnormal Runway Contact (ARC) and Runway Excursion (RE) holds the second place with controlled flight into terrain (CFIT) after the Loss-of-Control in Flight (LOC-I) [1]. Approach and landing accident reduction (ALAR) is the primary goal of the Flight Safety Foundation (FSF) [2]. It is noted that a better knowledge of flight dynamics in close proximity to the ground can provide increased understanding of the various crosswind handling techniques to increase safety during a crosswind landing [2].

Aircraft aerodynamic characteristics and dynamic behaviour are subjected to changes in proximity to the ground during landing approach and take-off flight [3]. An increase in the lift force, reduction in the amount of induced drag, onset of the pitching down moment requires control actions from the pilot for retrimming aircraft. The above mentioned aerodynamic changes due to ground effect in the aircraft longitudinal dynamics and control are well recognised. Special wind tunnel techniques are used for evaluation of the ground effect in the longitudinal aerodynamic characteristics [4]. An analytical study of the ground effect on the airplane longitudinal stability can be found, for example, in paper [5].

During crosswind landing and take-off the aircraft lateral-directional dynamics can be excited. Aircraft can be approaching and landing with sideslip and nonzero bank angle, this requires leveling aircraft in close proximity to the runway. Therefore the effect of closeness to the ground on the lateral-directional aerodynamic characteristics in such situations should be seriously evaluated. To the best knowledge of the authors, changes in the lateral-directional airplane dynamics due to ground effect have not been addressed in the aeronautical literature and not introduced in the flight simulation practice.

In this paper we approach the above problem by using CFD methods for computational prediction of airplane aerodynamic characteristics in static conditions, when the airplane is flying above the runway with nonzero bank angle. The Common Research Model (CRM) [6] [7] of a generic modern transport airplane was considered in its cruise configuration. The CFD simulations were conducted using ANSYS Fluent and OpenFOAM open source software [8]. Most of the previously reported CFD simulations of aerodynamics in ground effect have been carried out for two dimensional airfoils and low aspect ratio configurations [9] [10] [11] [12].

The ground effect in the CRM aerodynamic forces and moments dependencies has been identified in the CFD simulations and the obtained aerodynamic data were applied for stability and controllability analysis in the lateral-directional airplane motion. The performed dynamic analysis for a typical transport airliner showed transformation of the airplane lateral-directional modes of motion. For example, the roll subsidence and spiral eigenvalues in close proximity to the ground are merging creating the oscillatory Roll-Spiral mode with quite significant frequency. This transformation of the lateral-directional dynamics introduced in piloted simulation may affect the flight simulator motion-cueing and 
handling quality characteristics. The major factor of the performed ground effect dynamic analysis was the introduction of the rolling and yawing moments dependencies on the airplane bank angle, which was equivalent to the "aerodynamic banking stiffness". The airplane responses to ailerons and rudder control inputs also change in close proximity to the ground.

The formulation of the computational framework and simulation results for CRM ground effect aerodynamics are presented in Section 2. Additionally the preliminary results in grid generation for a high-lift configuration with fully deployed flaps and slats are discussed. Section 3 presents results of dynamic analysis for the lateral-directional motion and also the 6-DOF simulations of the full scale flight simulation model in close proximity to the ground.

\section{CFD Simulation of Ground Effect Aerodynamics}

For evaluation of the ground effect aerodynamics in this study the Common Research Model (CRM) geometry of a generic airliner was selected. The CRM geometry in wing-body-horizontal tail configuration is available on NASA repository for Drag Prediction Workshop [6]. The geometry was modified to include the ONERA vertical fin available at [7]. The reference area for CRM model, shown in Figure 1 is $S_{\text {ref }}=383.7 \mathrm{~m}^{2}$ and $C_{\text {ref }}=7 \mathrm{~m}$, the wing has an aspect ratio of $A R=9.0$.

\subsection{Grid Generation}

The build topology of the CRM model has been checked and corrected to ensure air tightness on the model surfaces. After this procedure a hexahedral mesh was generated for the full model. A structured mapped blocking approach with appropriate splits and inclusion of O-grids was used to better capture the boundary layer regions on the airplane surfaces.

The blocks initially generated, were transformed through rotations and translations to generate hexahedral unstructured meshes according to flight conditions, i.e. airplane attitude and closeness to the ground. The boundary conditions on the ground were implemented as a moving wall with direction and velocity magnitude of incoming flow and were resolved with inclusion of $\mathrm{H}$-grid layers with appropriate wall distance $(Y+<1)$.

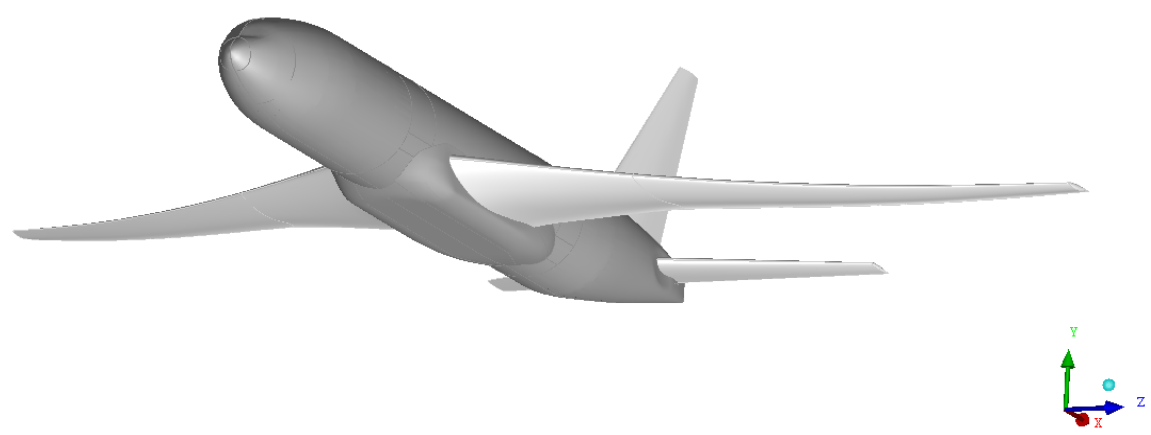

Figure 1. Full configuration of the Common Research Model (CRM). 
The initial meshes were generated for different altitudes above the ground, i.e. $h=4 \bar{c}, h=2 \bar{c}, h=1 \bar{c}$, and $h=0.5 \bar{c}$. At each altitude $h$, the grid was adapted for a number of different angles of attack settings $\alpha=4^{\circ}, 8^{\circ}, 12^{\circ}$. At altitude $h=0.5 \bar{c}$ additionally a number of bank angle settings was considered $\phi=4^{\circ}, 8^{\circ}, 12^{\circ}$ with additional adaptation of the grid. Figure 2 shows different CRM attitudes at $h=0.5 \bar{c}$ (different colors are used to highlight different aerodynamic surfaces of the model). The blue mesh in the bottom represents the ground.

The numerical simulations were carried out within reasonable accuracy of a grid between coarse to medium, i.e. about 10 million cells for a full body configuration. This seems suitable for our purpose here to evaluate ground effect.

\subsection{Governing Equations and Boundary Conditions}

The Navier-Stokes equations governing incompressible fluid flow are:

$$
\begin{gathered}
\nabla \cdot u=0 \\
\frac{\partial u}{\partial t}+(u \cdot \nabla) u-v \nabla^{2} u=-\nabla p / \rho
\end{gathered}
$$

For the Reynolds numbers typical for industrial applications, the computational resources required for a Direct Numerical Simulation (DNS) of Equations (1) (2) are exceeding the currently available technical capabilities. The effect of turbulence is normally simplified by solving the Reynolds-Averaged Navier-Stokes (RANS) equations, which are the time averaged approximation of Equations (1) (2). The averaging of fluctuating velocities generates additional terms, known as the Reynolds stresses. To describe these stresses the additional empirical equations, generally in the form of partial differential equations are required to close the computational model. The majority of RANS models are based on the concept of an eddy viscosity, equivalent to the kinematic viscosity of the fluid, which describes the turbulent mixing or the diffusion of momentum. For closure, in this study the turbulence $k$ - $\omega$-SST formulation is used [13].

$$
\frac{\partial k}{\partial t}+\frac{\partial\left(u_{j} k\right)}{\partial x_{j}}=P_{k}-\beta^{*} k \omega+\frac{\partial}{\partial x_{j}}\left(\mu+\sigma_{k} \mu_{t}\right) \frac{\partial \omega}{\partial t}
$$

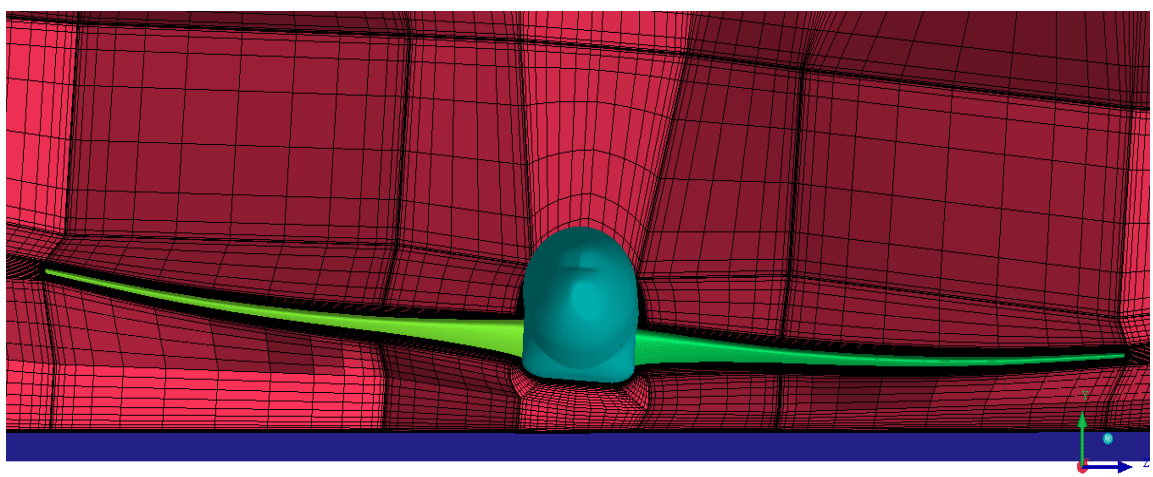

Figure 2. Generated meshes and flight settings. 


$$
\frac{\partial \omega}{\partial t}+\frac{\partial\left(u_{j} \omega\right)}{\partial x_{j}}=\frac{\gamma}{\mu_{t}} P_{k}-\beta \omega^{2}+\frac{\partial}{\partial x_{j}}\left(\mu+\sigma_{\omega} \mu_{t}\right)+D
$$

where turbulent viscosity is defined as:

$$
\mu_{t}=\frac{a_{1} k}{\max \left(a_{1} \omega, \Omega F_{2}\right)}
$$

Far field is assumed at least 100 chord lengths away from the aircraft in $\mathrm{x}$ and $y$ direction and $+z$ direction. The $-z$ distance was measured in terms of distance $h$ as it was normal to the ground. A free stream turbulence intensity $0.1 \%$ was assumed at the inlet and pressure was discretized to be zero gradient in normal direction at inlet, outlet and the airplane surfaces.

\subsection{Solver and Numerical Settings}

The ground effect aerodynamics was simulated using the steady-state and unsteady solver (U/RANS), closed by the $k$ - $\omega$-SST model for turbulence. Under relaxation is applied for solution of U/RANS equations to increase convergence stability. Second order discretization schemes were used to solve momentum and continuity equations. All scalar variables are solved with the first order accuracy. The residuals for all the equations are allowed to reach a satisfactory convergence of $1 / 10,000$ th of the initial values (see Figure 3 ).

In the case of unsteady simulations at moderately high angle of attack and bank angle, the steady state solution is computed first, which is then used to initialize the unsteady solution to improve the convergence and stability for the unsteady solution.

The simulations were run on a University cluster with 24 processors and the time for convergence for a single point in steady state simulations took an average of 1 - 2 days, while for unsteady simulations it took 4 - 5 days to reach convergence. The average CPU time was about $1.05 \mathrm{~min} /$ iteration. Most of the simulations ran from at least 5000 iterations to a maximum of 15,000 iterations.

The results for $M=0.4, R e=24$ million at zero angle of attack is validated against available data obtained at the Netherlands Aerospace Center (NLR)

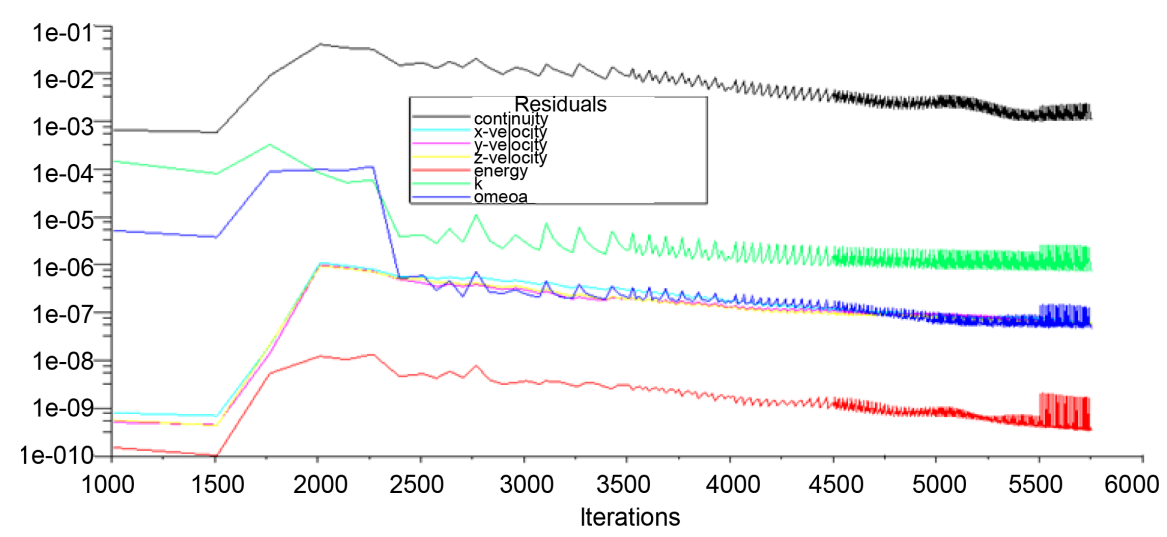

Figure 3. Convergence of residuals. 
using their in-house CFD code ENFLOW. The lift coefficient at the above mentioned flight conditions is $C_{L}=0.188$ for our simulations and $C_{L}=0.197$ for ENFLOW simulations. The variation in the lift coefficient between two codes is about $5 \%$ and is subject to many differences such as grid and numerical setup.

\subsection{Simulation Results}

In close proximity to the ground the airplane wing tip vortices are modified giving a reduced downwash contribution. This leads to increase in the lift force, reduction in the amount of induced drag, onset of the pitching down moment. For illustration purposes, Figure 4 shows a pressure distribution on the CRM surfaces, the ground and in a far field cross-section at flight with altitude $h=0.5 \bar{c}$, angle of attack $\alpha=8^{\circ}$ and bank angle $\phi=4^{\circ}$. Note, the wing closer to the ground has a much weaker tip vortex than a similar vortex on the upper wing, which appears to be slightly elliptical. There are also zones with increased pressure on the ground under the wing and horizontal tail.

Transformations of the wing tip vortices in ground effect produce changes in the aerodynamic forces and moments acting on the aircraft. Figure 5 presents simulated dependencies for the lift, drag and also for the pitching, rolling and yawing moment coefficients. The ground effect increments in the aerodynamic loads increase with increase of the magnitude of the lift and strengthening the wing tip vortices and downwash outside of the ground. For example, at $\alpha=8^{\circ}$ and zero bank angle $\phi=0^{\circ}$ the increase in the lift coefficient is $\Delta C_{L}=0.08$, which is equivalent to increase on $11.2 \%$ (see Figure 5, top left plot). Further increase in the lift coefficient takes place at bank angle $\phi=12^{\circ}, \Delta C_{L}=0.217$, which is equivalent to increase on $28.7 \%$ (see Figure 5 , top right plot). The drag coefficient at bank angle $\phi=12^{\circ}$ decreases, $\Delta C_{D}=-0.013$, this is equivalent to decrease on $11.8 \%$ (see Figure 5, top right plot).

The aerodynamic moments are also affected by the bank angle in close proximity to the ground. There is a significant pitching down effect at $\phi=12^{\circ}$, i.e. $\Delta C_{m}=-0.198$ (see Figure 5, bottom left plot). The most important for our objectives are the rolling and yawing moment dependencies on bank angle, shown in Figure 5, bottom right plot. The moment coefficient $C_{I}$ proportionally

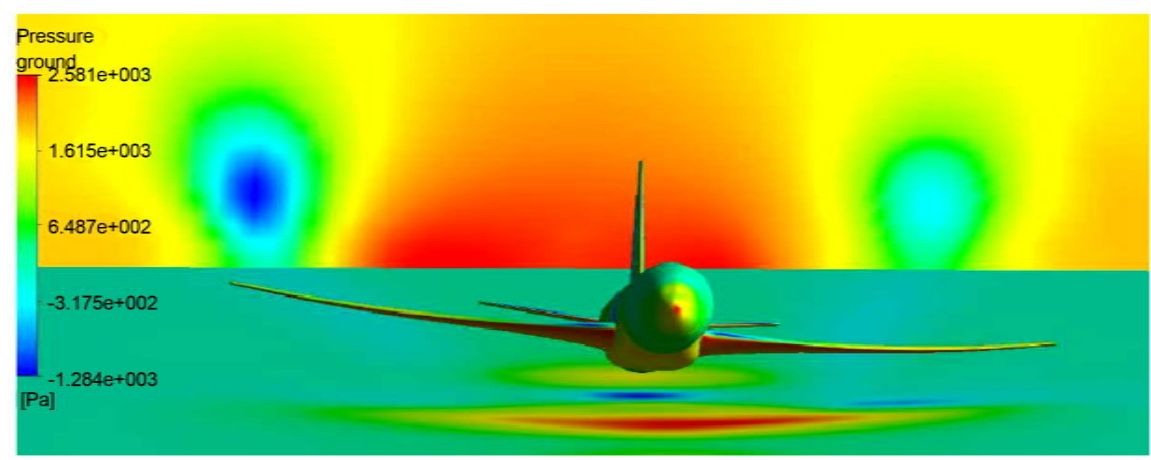

Figure 4. Pressure distribution on CRM surface, the ground and far field cross-section at $h=0.5 \bar{c}, \alpha=8^{\circ}$ and $\phi=4^{\circ}$. 

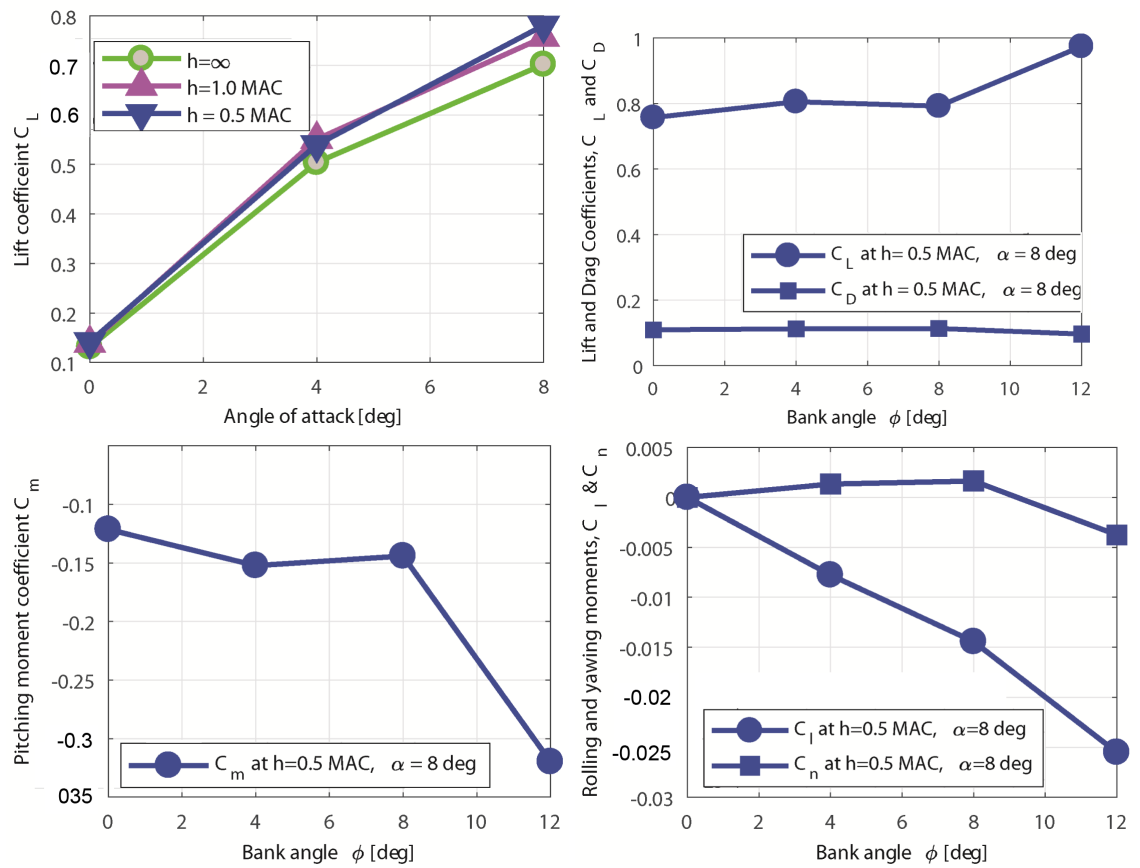

Figure 5. Effect of bank angle on aerodynamic coefficients $C_{L}, C_{D}, C_{m}, C_{p}, C_{n}$ at $h=0.5 \bar{c}, \alpha=8^{\circ}$.

decreases with increase of bank angle $\phi$, which is acting in a way as a stiff spring. The yawing moment coefficient increases with increase of bank angle until $\phi=8^{\circ}$, but decreases with the change of sign at $\phi=12^{\circ}$ (see Figure 5 , bottom left plot).

\subsection{High-Lift Configuration}

The ground effect in aerodynamic characteristics is proportional to the lift force. For CRM configuration we considered high angle of attack runway approach. The airplanes are normally approach landing with deployed leading and trailing edge flaps, which produce a high lift at low angles of attack. Hereafter, we present preliminary set up for a high-lift configuration.

\subsubsection{Geometry}

A hybrid model is constructed by combining the wing-body configuration (DLR F11) provided in the 2nd AIAA High Lift Prediction workshop [14] and the NASA General Transport Model (GTM) available in Open VSP Hangar [15]. This is successfully done by trimming the vertical and horizontal stabilizers along with fuselage rear end at roughly $34^{\text {th }}$ of the fuselage length from the nose. The geometry is then also simplified for CFD by cleaning up in terms of topology. Surfaces are further simplified and merged together to automate the ease of blocking with caution such that the authenticity of the model is not lost and the geometry is not violated.

The reference area for this model is $419,130 \mathrm{~mm}^{2}$ for the half model and the mean aerodynamic chord length of $347.09 \mathrm{~mm}$ is at quarter chord along wing 
span. The flaps are deflected down at 32 degrees and the slats are deflected down at 26.5 degrees.

\subsubsection{Grid Generation}

The grid for this particular configuration is structured using Hexa- 8 and quad- 4 elements. The hexa elements are ideally 8 node elements in 3D space, and 2D quads are 4 node elements. Such a grid is made using blocking and mapping the blocks to the model under consideration. The current mesh contains more than 1500 blocks and hence for complex full flight configurations such as DLR F11 it is difficult to maintain mesh quality in terms of orthogonality, skewness and aspect ratio for such a mesh. This becomes even more difficult when specially applied to the small gaps in between the flaps, slats and the main wing as we need to resolve the boundary layer for each of them separately, but also maintain connectivity in mesh such that they are resolved as a single structure.

However, as seen in Figure 6 and Figure 7 the special blocking allows us to control the boundary layer from flaps, slats and wing without having them collapse each other. This is one of the main advantages of using a structured blocking approach for such configurations along with other benefits such as reduction in cell count, higher quality meshes and more flexible and solvable by matrix solvers as the nodes are in a much regular order.

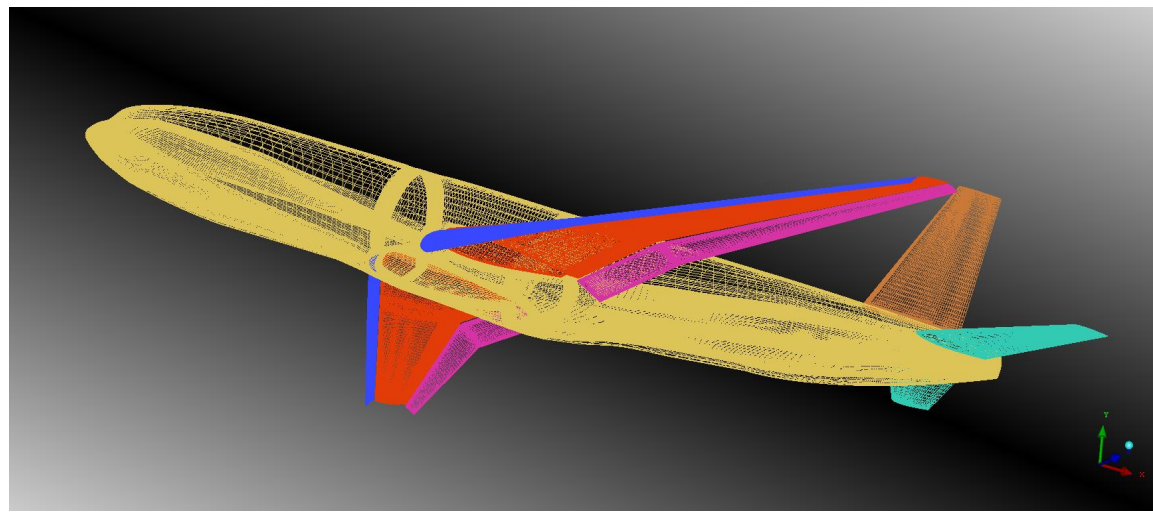

Figure 6. Isometric wireframe view of the surface grid for F11-GTM model.

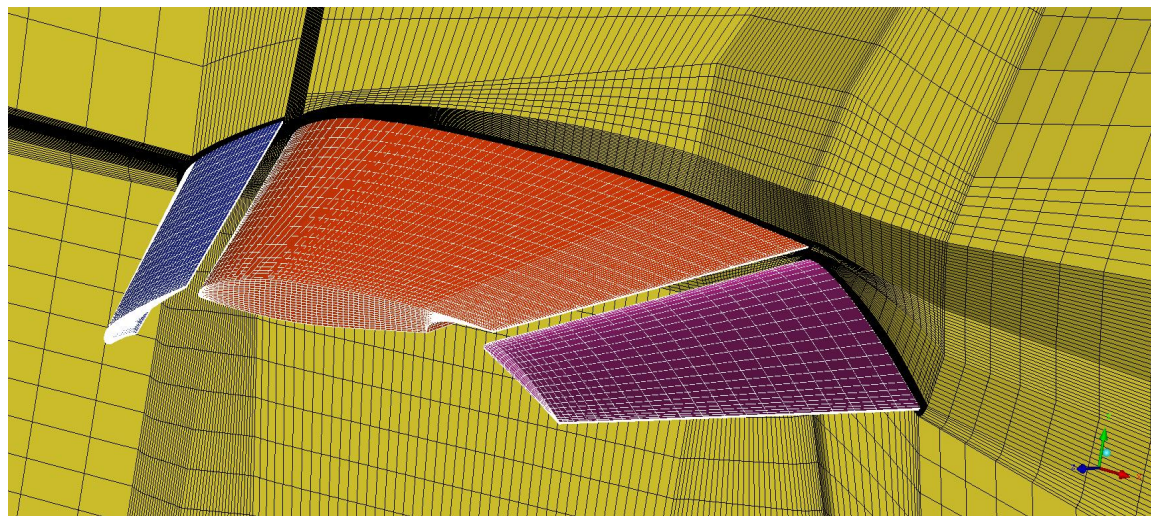

Figure 7. Scan plane through volume mesh for the F11-GTM model. 
The gridding also follows the guide lines given by the High Lift Prediction Workshop [14]. A coarse mesh according to [14] should have the wall spacing of $\mathrm{Y}+=1$ and in dimensional form is $\mathrm{y}=0.00055 \mathrm{~mm}$. With the increase in mesh size a proportional decrease in wall distance y, should be implemented. It is also advised that at least the first 2 layers in the boundary should have a constant cell spacing. A minimum of at least 4 cells is recommended in the trailing edge for a coarse mesh and the boundary growth is to be at the ratio of $<1.25$. The spacings should be roughly $0.1 \%$ of the span wise length and chord wise local length parallel to the direction of the grid. Furthermore, as common practice indicates, the far field is at least $100 C_{\text {ref }}$ away from the body in every direction. The grids are intended for the landing conditions of $R e=15.1$ million and the same grids are recommended to be used for lower Reynolds tests as well.

Future plans include to run CFD simulations for the ground effect aerodynamics considering the described high-lift configuration with additional objective to evaluate unsteady and rotary aerodynamic derivatives in proximity to the ground and their effect on the lateral-directional stability.

\section{Airplane Lateral-Directional Dynamics in Close Proximity to the Ground}

The obtained in CFD simulations dependencies for the aerodynamic coefficients, presented in the previous section, have been used for modification of the full flight simulation model of a typical transport aircraft for conducting 6-DOF simulations in a level trimmed flight in close proximity to the ground. Trim and linearisation procedures have been applied to evaluate aircraft stability conditions and small amplitude modes of motion in the longitudinal and lateral-directional motion. Additionally, the impact of ground proximity on stability of the lateral-direction dynamics is addressed in this section complemented by the eigenvalues analysis for the linearised lateral-directional equations.

\subsection{Lateral-Directional Equations}

For evaluation of the airplane lateral-directional dynamics in close proximity to the ground the stability-axis lateral-directional equations are considered in the following vector-matrix form (see notations in [16]):

$$
\left.\left|\begin{array}{c}
\dot{r} \\
\dot{\beta} \\
\dot{p} \\
\dot{\phi}
\end{array}\right|=\left[\begin{array}{cccc}
N_{r} & N_{\beta} & N_{p} & N_{\phi} \\
-1 & \bar{Y}_{\beta} & 0 & g / V \\
L_{r} & L_{\beta} & L_{p} & L_{\phi} \\
0 & 0 & 1 & 0
\end{array}\right]\left|\begin{array}{l}
r \\
\beta \\
p \\
\phi
\end{array}\right|+\left[\begin{array}{ll}
N_{\delta_{a}} & N_{\delta_{r}} \\
0 & 0 \\
L_{\delta_{a}} & L_{\delta_{r}} \\
0 & 0
\end{array}\right] \mid \begin{array}{l}
\delta_{a} \\
\delta_{r}
\end{array}\right]
$$

The new terms in the state matrix of Equation (6) are $N_{\phi}=C_{n \phi}(\bar{h}) \frac{\rho V^{2} S b}{2 I_{z z}}$ and $L_{\phi}=C_{l \phi}(\bar{h}) \frac{\rho V^{2} S b}{2 I_{x x}}$. They represent the rolling and yawing accelerations induced by bank angle $\phi$. The ground effect in this case is equivalent to a kind of 
"aerodynamic roll stiffness", which will tend to level the airplane above the runway.

In flight away from the ground, when $N_{\phi}=L_{\phi}=0$, the lateral-directional modes are defined by the Roll-Dutch complex-conjugate eigenvalues $\lambda_{\mathrm{DR}}=-\xi \pm \omega_{n} \sqrt{1-\xi^{2}}$, the roll subsidence eigenvalue $\lambda_{R}$ and the spiral mode eigenvalue $\lambda_{S}$. It is reasonable to represent the ground effect in the form of a root-loci with a parameter indicating variation of the reduced flight altitude $\bar{h}=h / \bar{c}$.

\subsection{Oscillatory Roll-Spiral Mode in Lateral-Directional Dynamics}

The lateral-directional characteristic equation with account of ground effect can be represented in the following form:

$$
\left(s-\lambda_{s}\right)\left(s-\lambda_{R}\right)\left(s^{2}+2 \xi \omega_{n}+\omega_{n}^{2}\right)_{D R}-L_{\phi}\left(s^{2}+a_{1} s+a_{0}\right)_{G E}=0
$$

where

$$
\begin{gathered}
a_{1}=\frac{N_{\phi}}{L_{\phi}} L_{r}-N_{r}-\bar{Y}_{\beta} \\
a_{0}=N_{\beta}-\frac{N_{\phi}}{L_{\phi}} L_{\beta}+\left(N_{r}-\frac{N_{\phi}}{L_{\phi}} L_{r}\right) \bar{Y}_{\beta}
\end{gathered}
$$

Parameter $L_{\phi}$ varies from zero value in flight with no ground effect $(\bar{h}=\infty)$ to its maximum value in close proximity to the ground $(\bar{h}=0.5-1.0)$. The $N_{\phi} / L_{\phi}$ ratio in expressions $a_{1}$ and $a_{2}(8)$ has a weak dependence on reduced altitude $\bar{h}$. So, with increase of parameter $L_{\phi}$ the eigenvalues will move on the complex plane from their initial values $\lambda_{D R}=-\xi \pm \omega_{n} \sqrt{1-\xi^{2}}, \lambda_{R}$ and $\lambda_{S}$ toward $\mathrm{s}$ the values defined by zeros $z_{1}, Z_{2}$ of the second order polynomial equation $s^{2}+a_{1} s+a_{0}=0$ and one pair of eigenvalues will migrate to infinity. The location of zeros $z_{1}$ and $z_{2}$ depends on lateral directional coefficients in the expressions for $a_{1}$ and $a_{0}(8)$. These zeros can be located in the left half of the complex plane, being a complex-conjugate pair, or move to the right unstable half of the complex plane creating an opportunity for onset of oscillatory instability due to ground effect, when $a_{1}<0$. There is also a possibility for onset of aperiodical instability due to ground effect if $a_{0}<0$.

A full flight simulation model of a typical transport aircraft has been modified taking into account the aerodynamic dependencies presented in the previous section for the 6-DOF flight simulations in a level trimmed flight in close proximity to the ground. The ground effect is strong in close proximity to the ground and at the altitude exceeding four mean aerodynamic chords practically vanishes.

The eigenvalues of the linearised equations of motion are presented in Figure 8 with variation of parameter $\bar{h}$. The eigenvalues root-loci shows significant transformation of the lateral-directional modes of motion.

The roll subsidence and spiral eigenvalues in close proximity to the ground 

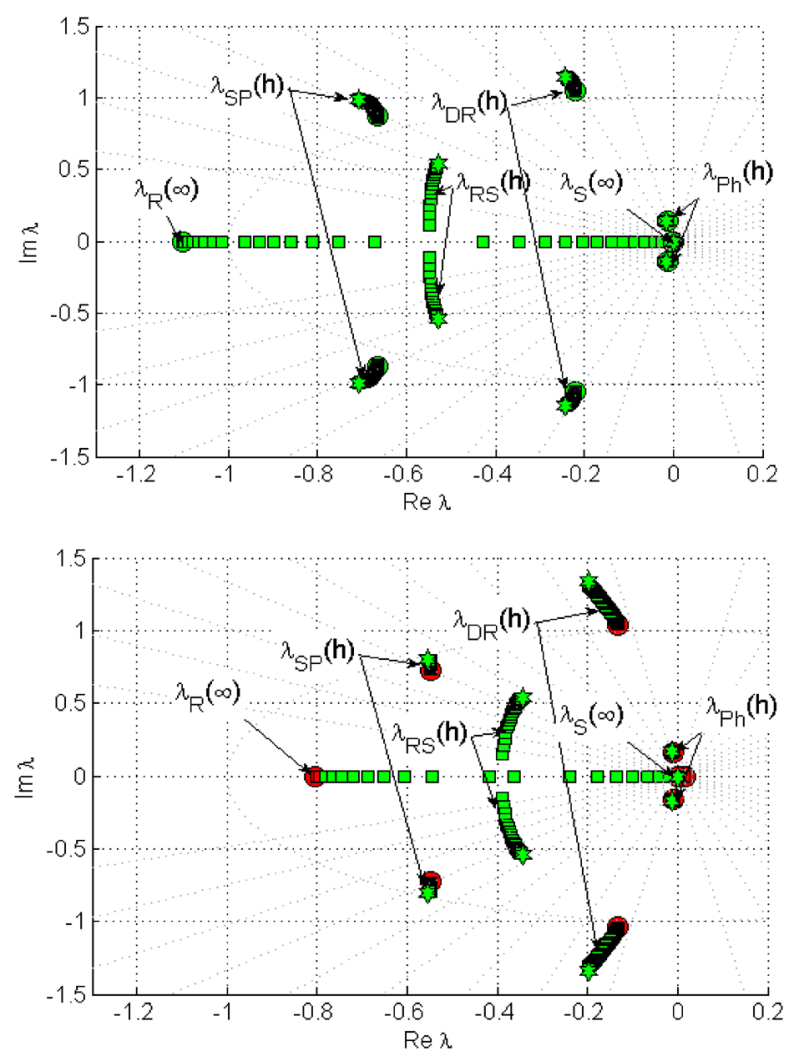

Figure 8. Root-loci of eigenvalues of the longitudinal and lateral-directional dynamics modes at different altitudes above the runway $\bar{h}=4 \bar{c}, \bar{h}=2.5 \bar{c}$ and $\bar{h}=1 \bar{c}$, top plot- $\alpha=3^{\circ}$ and bottom plot $\alpha=8^{\circ}$.

are merging creating the oscillatory Roll-Spiral mode with quite significant frequency $\omega_{R S}=0.538 \mathrm{rad} / \mathrm{s}$ (see Figure 8, bottom plot, $\alpha=8^{\circ}$ ). Along with this change, the Dutch-roll eigenvalues increase frequency from the level of $\omega_{D}=1.05 \mathrm{rad} / \mathrm{s}$ to $\omega_{D}=1.34 \mathrm{rad} / \mathrm{s}$.

There are very little changes in the short-period longitudinal eigenvalues, $\lambda_{S P}$ and practically no changes in the longitudinal phugoid mode, $\lambda_{P h}$. In Table 1 the eigenvalues for the lateral-directional motion modes for flight at $h=\infty$ and $\bar{h}=1 \bar{c}$, are presented for clarity showing a substantial transformation of the lateral-directional dynamics.

The new factor introduced in the performed eigenvalues analysis was the rolling and yawing moments depending on the airplane bank angle, which was equivalent to the "aerodynamic banking stiffness". This "aerodynamic stiffness" is strongly affecting the airplane controllability in close proximity to the ground. The airplane responses to aileron and rudder control inputs obtained in the 6-DOF flight simulation are shown in Figure 9 and Figure 10, respectively. The airplane responses to pilot control inputs change in amplitudes and frequencies at low reduced altitude $\bar{h}=h / \bar{c}$. This may lead to changes in handling qualities at low altitudes with effect on crosswind landing and onset of pilot induced oscillations. 

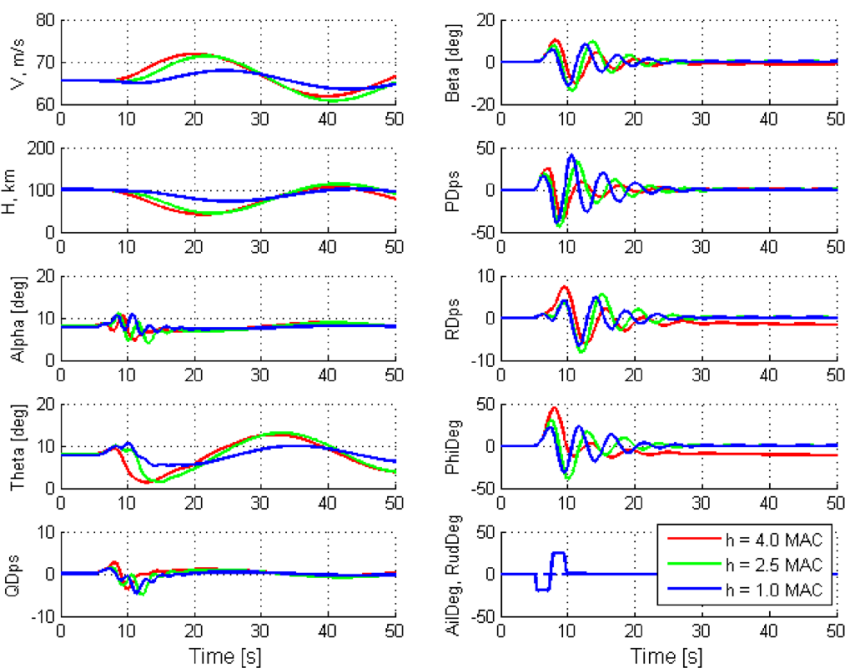

Figure 9. Airplane responses to doublet aileron control input $\delta_{a}= \pm 25^{\circ}$ at different altitudes above the runway obtained in 6-DOF simulation.
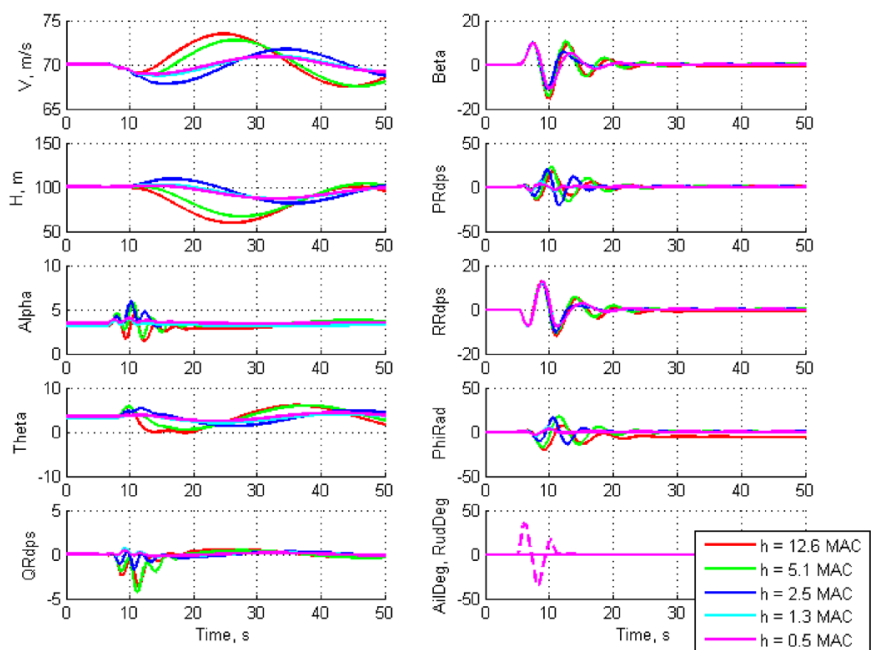

Figure 10. Airplane responses to doublet rudder control input $\delta_{r}= \pm 35^{\circ}$ at different altitudes above the runway obtained in 6-DOF simulation.

Table 1. Eigenvalues of Longitudinal and Lateral-Directional Dynamics Modes at $\alpha=8^{\circ}$.

\begin{tabular}{cccccc}
\hline $\begin{array}{c}\text { Ground } \\
\text { Effect }\end{array}$ & Short Period (SP) & Phugiod (Ph) & Roll $^{\odot}$ & Spiral (S) & Dutch Roll (DR) \\
\hline$h(\infty)$ & $0.549 \pm 0.742 \mathrm{i}$ & $0.012 \pm 0.17 \mathrm{i}$ & -0.8 & -0.008 & $-0.133 \pm 1.048 \mathrm{i}$ \\
$h=0.5 \bar{c}$ & $-0.554 \pm 0.8 \mathrm{i}$ & $-0.012 \pm 0.17 \mathrm{i}-0.344+0.538 \mathrm{i}$ & $-0.344-0.538 \mathrm{i}$ & $-0.2 \pm 1.335 \mathrm{i}$ \\
\hline
\end{tabular}

In crosswind approach-and-landing the aircraft should fly with some nonzero sideslip angle to compensate side-wind. To fly a straight line along the runway the aircraft at the same time should have some non-zero sideslip and bank 


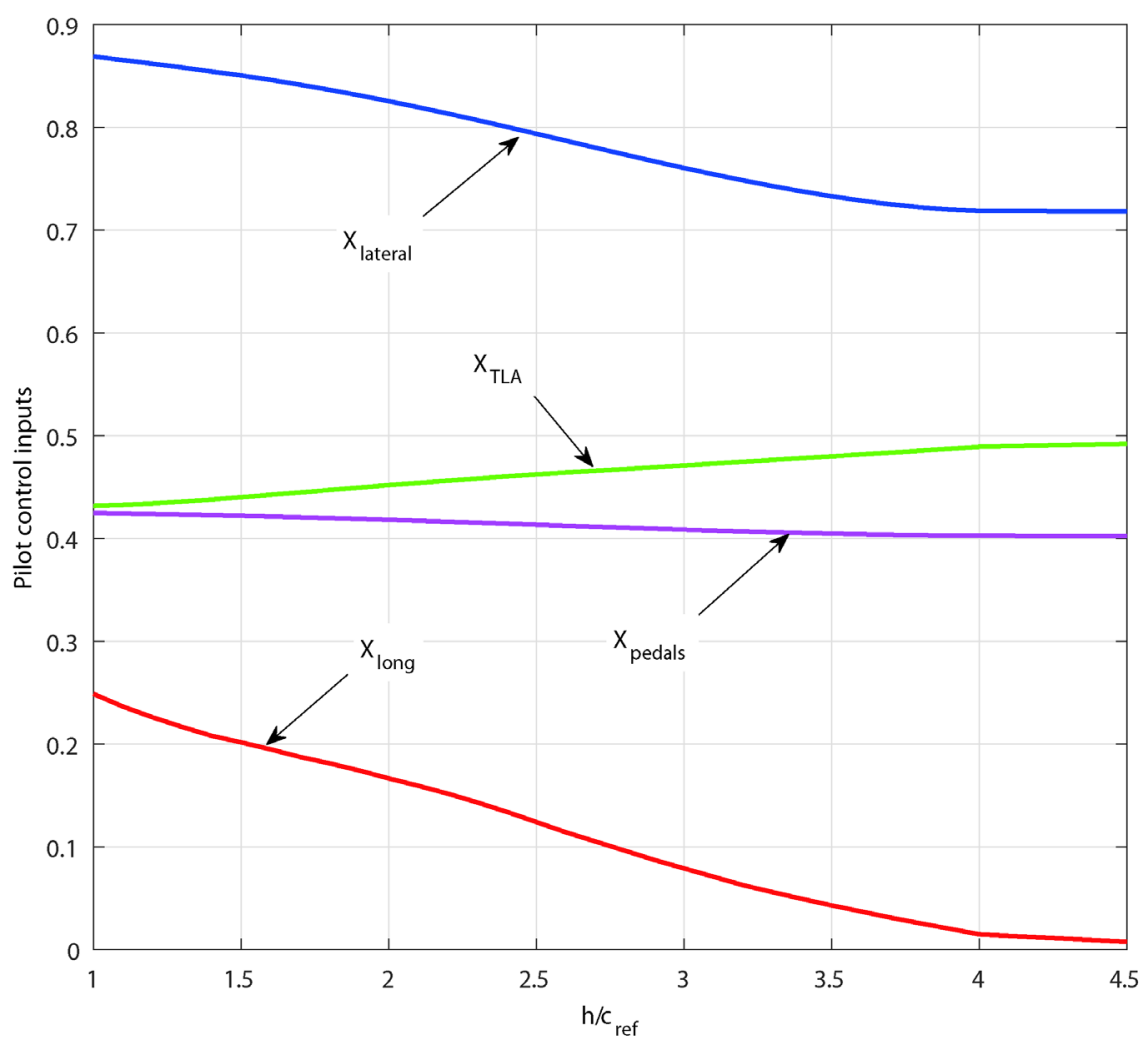

Figure 11. Trim control inputs required in landing approach with nonzero sideslip and bank angles $\left(\alpha=8^{\circ}, \beta=10^{\circ}, \phi=4^{\circ}\right)$.

angles. Figure 11 shows the required control pilot inputs in trim flight with steady sideslip $\alpha=8^{\circ}, \phi=4^{\circ}$ and $\beta=10^{\circ}$. The control inputs are normalised with respect to maximum deflections. One can see that during landing significant retrimming is required in the longitudinal and lateral control channels and thrust control, and less sensitivity is shown in the directional channel.

\section{Concluding Remarks}

CFD simulation results for evaluation of the ground effect aerodynamics have been obtained for CRM model [17] in its cruise configuration using Fluent software. The k- $\omega$ SST turbulence model and the "moving wall" boundary conditions were utilised to realistically simulate runway boundary layer. The future plans include evaluation of the ground effect aerodynamics for a high-lift configuration F11/GTM using the OpenFOAM software with objective to additionally evaluate unsteady and rotary aerodynamic derivatives in close proximity to the ground and their effect on the lateral-directional stability.

The presented dynamic analysis of the lateral-directional motion modes and controllability during approach-and-landing shows the importance of the ground effect for the improved realism of piloted simulation and estimation of critical crosswinds. The introduced aerodynamic modelling allows improved pilot training on various types of flight simulators. 


\section{Acknowledgements}

The authors appreciate discussions with test pilot Vladimir Biryukov and are grateful for his comments on crosswind approach-and-landing. His participation in preliminary piloted simulations helped to tune the aerodynamic model to produce realistic results.

\section{References}

[1] Statistical Summary of Commercial Jet Airplane Accidents (2015) World Wide Operations 1959-2015. Aviation Safety, Boeing Commercial Airplanes, 2015.

[2] Flight Safety Foundation (2000) Approach-and-Landing Accident Reduction (ALAR) Briefing Note: 8.7-Crosswind Landing. Flight Safety Foundation, Flight Safety Digest. https://flightsafety.org/files/alar_bn8-7-crosswind.pdf

[3] Cui, E. and Zhang, X. (2010) Ground Effect Aerodynamics. In: Encyclopedia of Aerospace Engineering, Wiley Online Library. https://doi.org/10.1002/9780470686652.eae022

[4] Meredith, C. and Evans,W. (2000) Ground Effect Testing Capabilities in the Filton $12 \times 10$ Low Speed Wind Tunnel. 22nd International Congress of the Aeronautical Sciences, Harrogate, 27 August-1 September 2000, Paper ICAS-373.

[5] Staufenbiel, R.W. and Schlichting, U.-J. (1988) Stability of Airplanes in Ground Effect. Journal of Aircraft, 25, 289-294. https://doi.org/10.2514/3.45562

[6] https://aiaa-dpw.larc.nasa.gov/Workshop5/DPW5-geom.html

[7] https://commonresearchmodel.larc.nasa.gov/geometry/vertical-tail\%20geometry/

[8] OpenFOAM (2017) The Open Source Computational Fuid Dynamics Toolbox. http://www.openfoam.com/

[9] Ahmed, M.R., Takasaki, T. and Kohama, Y. (2007) Aerodynamics of a NACA 4412 Airfoil in Ground Effect. AIAA Journal, 45, 37-47. https://doi.org/10.2514/1.23872

[10] Doig, G. and Barber, T.J. (2011) Considerations for Numerical Modeling of Inverted Wings in Ground Effect. AIAA Journal, 49, 2330-2333. https://doi.org/10.2514/1.J051273

[11] Mahon, S. and Zhang, X. (2005) Computational Analysis of Pressure and Wake Characteristics of an Aerofoil in Ground Effect. Journal of Fluids Engineering, Transactions of the ASME, 127, 290-298. https://doi.org/10.1115/1.1891152

[12] Zhang, X. and Zerihan, J. (2004) Edge Vortices of a Double-Element Wing in Ground Effect. Journal of Aircraft, 41, 1127-1137. https://doi.org/10.2514/1.1380

[13] Menter, F.R. (1992) Improved Two-Equation $k$ - $\omega$ Turbulence Models for Aerodynamic Flows. TM 103975, NACA.

[14] 2nd AIAA CFD High Lift Prediction Workshop (2017). https://hiliftpw.larc.nasa.gov/index-workshop2.html

[15] OpenVSP Sofware and Hangar (2017). http://hangar.openvsp.org/ http://openvsp.org/

[16] Robert, F. (2004) Stengel. Flight Dynamics. Princeton University Press, Princeton.

[17] Abramov, N.B., Sereez, M. and Goman, M.G. (2017) Computational Ground Effect Aerodynamics and Airplane Stability Analysis during Take-Off and Landing. EUCASS Conference Paper, 7 th European Conference for Aeronautics and Space Sciences, Milan, July 2017. 